\title{
Radio-frequency driven polarization transfer without heteronuclear decoupling in rotating solids is
}

\author{
Colan E. Hughes, Sorin Luca ${ }^{1}$, Marc Baldus * \\ Department of NMR Based Structural Biology, Max-Planck Institute for Biophysical Chemistry, Am Faßberg 11, 37077 Göttingen, Germany
}

Received 29 August 2003; in final form 7 January 2004

Published online: 29 January 2004

\begin{abstract}
This Letter reports significant excitation of ${ }^{13} \mathrm{C}$ homonuclear double-quantum coherence in a solid-state magic-angle-spinning NMR experiment, acquired without heteronuclear decoupling of abundant ${ }^{1} \mathrm{H}$ spins. The heteronuclear decoupling efficiency of a group of existing homonuclear polarization transfer sequences are investigated and optimal schemes are identified. Since the approach permits the application of fast MAS and significantly reduces the total radio-frequency power used, it is particularly well suited to biomolecular applications at ultra-high magnetic fields.
\end{abstract}

(C) 2004 Elsevier B.V. All rights reserved.

\section{Introduction}

Radio-frequency (r.f.) driven polarization transfer is of central importance in many high-resolution (i.e., magic angle spinning, MAS [1]) solid-state NMR experiments. Pulse schemes that enhance spin-spin interactions are used throughout the structural characterization of biomolecules, from the determination of individual distances [2-5] and torsional angles [6-8] to spectral assignment and structural characterization in multiply labelled biomolecules [9-11]. While significant progress has been made in optimizing the efficiency and character of the polarization transfer between two isotope-labelled rare spins, the empirically determined transfer efficiencies frequently fall considerably below the theoretically expected maxima. Often, these discrepancies are related to the presence of strong heteronuclear couplings and homonuclear couplings to surrounding protons that compromise the transfer efficiency.

\footnotetext{
Presented in part at the 25th GDCh Magnetic Resonance Meeting, 29th September-2nd October 2003, Leipzig, Germany.

${ }^{*}$ Corresponding author. Fax: +495512012202.

E-mail address: maba@mpibpc.mpg.de (M. Baldus).

${ }^{1}$ Present address: Laboratory of Molecular Biology, NIDDK, NIH, Bethesda, MD 20892-8030, USA.
}

Ishii et al. [12] investigated ${ }^{13} \mathrm{C}-{ }^{1} \mathrm{H}$ interactions under the application of both r.f. driven polarization transfer and ${ }^{1} \mathrm{H}$ decoupling schemes in solid-state NMR. From theory and experimental observations, they concluded that, for the case of static, windowed transfer sequences, a ratio for the nutation frequencies of the ${ }^{1} \mathrm{H}\left(\omega_{1 \mathrm{H}}\right)$ and ${ }^{13} \mathrm{C}\left(\omega_{1 \mathrm{C}}\right)$ nuclei of three to one was desirable to avoid loss of ${ }^{13} \mathrm{C}-{ }^{13} \mathrm{C}$ polarization transfer efficiency due to ${ }^{13} \mathrm{C}-{ }^{1} \mathrm{H}$ interactions. They also observed experimentally that similar sequences only required a value of $\omega_{1 \mathrm{H}} / \omega_{1 \mathrm{C}}=2.5$ under MAS conditions. Since then, a value of $\omega_{1 \mathrm{H}} / \omega_{1 \mathrm{C}}=2.5-3$ has generally been taken as optimal, combining good decoupling with minimal power levels. However, with recoupling schemes such as the $\mathrm{C}$ sequences [13], in which the rare spins are permanently irradiated at a nutation frequency proportional to the MAS frequency, this condition can be difficult to fulfil.

For polarization transfer at higher spinning frequencies, experiments have been developed in which the ratio of recoupling nutation frequency to MAS frequency is low [14,15]. In addition, dipolar recoupling using an adiabatic modulation of the system Hamiltonian has been demonstrated using no [16] or small [17] r.f. fields on the recoupling channel. Although these methods reduce the r.f. power necessary at high MAS frequencies, they may suffer from a high 
sensitivity to isotropic or anisotropic chemical shielding interactions or they may not be readily applicable in the context of multi-dimensional multiple-quantum spectroscopy.

Many of the well-performing dipolar recoupling methods also suppress anisotropic chemical shielding and heteronuclear dipolar interactions. Hence, it should be possible to address both aspects with the same polarization transfer scheme. We will show that the selection of a pulse scheme well optimized for the suppression of heteronuclear dipolar interactions can remove the necessity of ${ }^{1} \mathrm{H}$ decoupling during homonuclear double-quantum excitation and reconversion, without compromising the double-quantum filtering efficiency. Although we will only consider the case of homonuclear dipolar recoupling between two ${ }^{13} \mathrm{C}$ spins, the theoretical principles are valid for application to heteronuclear spin species or can be modified for the case of scalar through-bond transfer where heteronuclear (solution-state NMR) decoupling sequences have previously been used to establish through-bond polarization transfer in rotating solids [18].

\section{Theory}

Assuming a general $(\mathrm{I}, \mathrm{S})$ spin system, we have examined several $\mathrm{C}$ sequences [13] as candidates for (S,S) polarization transfer without heteronuclear (I) decoupling. From the symmetry theorem of $\mathrm{C}$ sequences, selection rules can be constructed. Following the notation of Levitt et al., the selection rule for the first-order average Hamiltonian [19] terms is

$\bar{H}_{l, m, \lambda, \mu}^{(1)}=0 \quad$ if $m n-\mu \nu \neq N Z$,

where the sequence symmetry is $\mathrm{CN}_{n}^{v}, l$ and $m$ are the space rank and component, and $\lambda$ and $\mu$ are the spin rank and component. For double-quantum homonuclear recoupling, terms with either $\{l, m, \lambda, \mu\}=\{2, \pm 1$, $2, \pm 1\}$ or $\{2, \pm 1,2, \mp 1\}$ are required. These retain homonuclear first-order dipolar terms whilst suppressing the other interactions to first-order. $J$-couplings and isotropic chemical shifts are not removed by these symmetries. A table of symmetries that select these terms is given in [13].

Of these symmetries, $\mathrm{C} 7_{2}^{1}[6]$ is the most commonly used, as in POST-C7 [20] where the $\mathrm{C}$ element is $90_{0} 360_{180} 270_{0}$ (known as the POST element). This element is robust towards chemical shift offsets and is preferred for double-quantum homonuclear recoupling. Alongside $\mathrm{C} 72$, we have investigated a number of other $\mathrm{CN}_{n}^{v}$ symmetries, listed in Fig. 1, first by calculating the desired first-order homonuclear dipolar term $\left(\bar{H}_{\mathrm{D}, \mathrm{SS}}^{(1)}\right)$ and the undesired second-order heteronuclear dipolar term $\left(\bar{H}_{\mathrm{D}, \mathrm{IS}}^{(2)}\right)$. The magnitude of $\bar{H}_{\mathrm{D}, \mathrm{SS}}^{(1)}$ is given by the scaling factor, $\kappa$, defined by
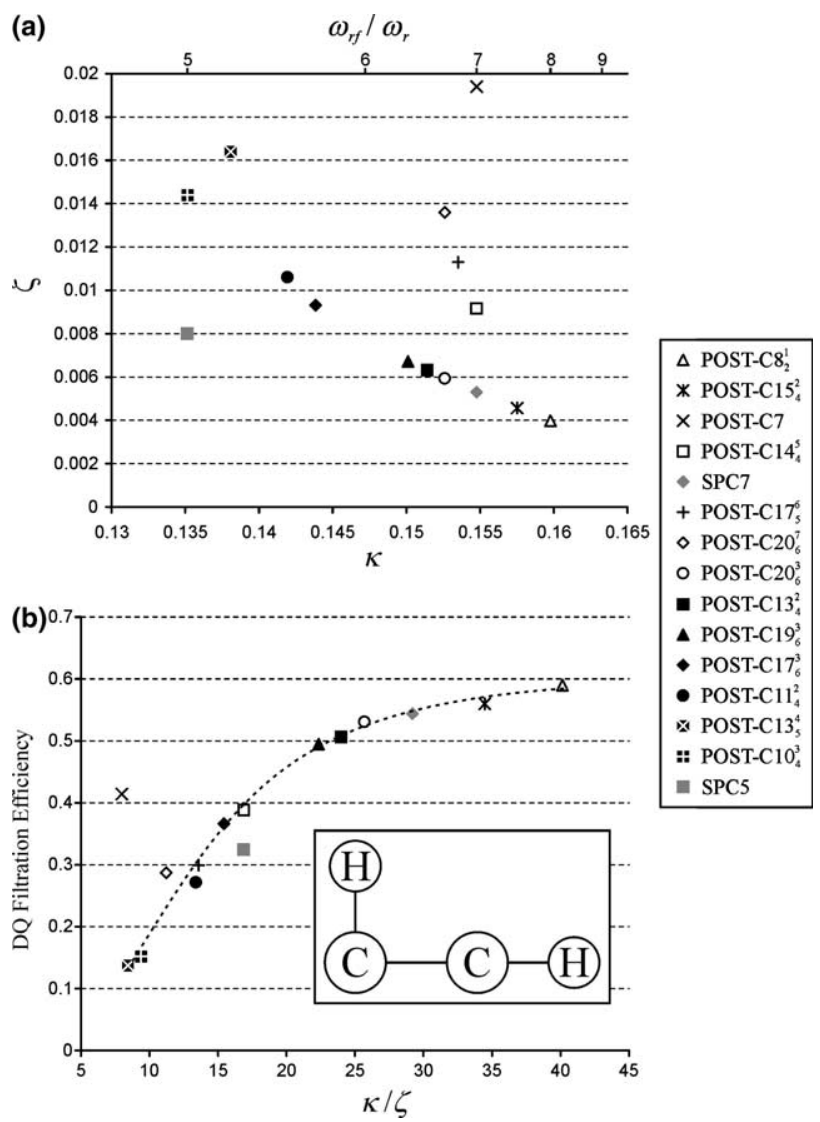

Fig. 1. (a) The powder average of the magnitude of the second-order heteronuclear dipolar average Hamiltonian, $\zeta$, plotted against the homonuclear recoupling scaling factor $\kappa$ (lower scale) and the r.f. nutation frequency divided by the MAS frequency, $\omega_{\mathrm{rf}} / \omega_{\mathrm{r}}$ (upper scale) for several pulse sequences. (b) Maximum simulated ${ }^{13} \mathrm{C}_{2} 2 \mathrm{QF}$ efficiencies for a ${ }^{1} \mathrm{H}-{ }^{13} \mathrm{C}-{ }^{13} \mathrm{C}-{ }^{1} \mathrm{H}$ spin system, as described in the text, of the same sequences, plotted against $\kappa / \zeta$. The simulation parameters are given in the text. The dashed curve is drawn as an aid to the eye.

$\bar{H}_{\mathrm{D}, \mathrm{SS}}^{(1)}=\left(\kappa^{-\mathrm{i} \gamma_{\mathrm{PR}}} T_{2,2}^{\mathrm{SS}}+\kappa^{*} \mathrm{e}^{\mathrm{i} \gamma_{\mathrm{PR}}} T_{2,-2}^{\mathrm{SS}}\right) b_{\mathrm{SS}} \sin \left(2 \beta_{\mathrm{PR}}\right)$,

where $b_{\mathrm{SS}}$ is the homonuclear dipolar coupling constant and $\beta_{\mathrm{PR}}$ and $\gamma_{\mathrm{PR}}$ are Euler angles relating the principal axis system of the dipolar interaction to the rotor frame.

To count the number of allowed $\bar{H}_{\mathrm{D}, \mathrm{IS}}^{(2)}$ terms, the second-order selection rules may be employed [13]. These are

$\bar{H}_{l_{1}, m_{1}, \lambda_{1}, \mu_{1}, l_{2}, m_{2}, \lambda_{2}, \mu_{2}}^{(2)}=0 \quad$ if $\left\{\begin{array}{l}m_{1} n-\mu_{1} v \neq N Z, \\ m_{2} n-\mu_{2} v \neq N Z, \\ \left(m_{1}+m_{2}\right) n-\left(\mu_{1}+\mu_{2}\right) v \neq N Z .\end{array}\right.$

For all the sequences investigated, we have found that the same eight terms are allowed, with only $\mathrm{C} 7{ }_{2}^{1}$ and $\mathrm{C} 8_{2}^{1}$ permitting additional terms ( 12 and four terms, respectively). To assess the actual magnitude of the secondorder heteronuclear dipolar term, we calculated the term $\bar{H}_{\mathrm{D}, \mathrm{IS}}^{(2)}$, defined as 
$\bar{H}_{\mathrm{D}, \mathrm{IS}}^{(2)}=\int_{t^{0}}^{t^{0}+n \tau_{\mathrm{r}}} \int_{t^{0}}^{t_{2}}\left[H_{\mathrm{D}, \mathrm{IS}}\left(t_{1}\right), H_{\mathrm{D}, \mathrm{IS}}\left(t_{2}\right)\right] \mathrm{d} t_{1} \mathrm{~d} t_{2}$,

using the semi-continuous Baker-Campbell-Hausdorff expansion [20,21], with the average being taken over $n$ rotor periods, i.e., over the repeating unit of the sequences. We then calculated the powder average of the magnitude of this term (removing the factor $b_{\mathrm{IS}}^{2} / \omega_{\mathrm{r}}$ )

$$
\begin{aligned}
\zeta= & \frac{\omega_{\mathrm{r}}}{b_{\mathrm{IS}}^{2}} \int_{0}^{2 \pi} \int_{0}^{\pi} \operatorname{Tr}\left\{\bar{H}_{\mathrm{D}, \mathrm{IS}}^{(2)} \bar{H}_{\mathrm{D}, \mathrm{IS}}^{(2)}\right\}^{1 / 2} \\
& \times \sin \left(\beta_{\mathrm{PR}}\right) \mathrm{d} \beta_{\mathrm{PR}} \mathrm{d} \gamma_{\mathrm{PR}},
\end{aligned}
$$

approximated by summing over a set of 50 angles generated by the ZCW algorithm [22]. It should be noted that $\zeta$ has no direct physical meaning and is used here as an approximate measure of the size of $\bar{H}_{\mathrm{D} \text { IS }}^{(2)}$, independent of the MAS frequency $\left(\omega_{\mathrm{r}}\right)$ and the dipolar coupling constant, since a scaling factor cannot be defined in the same way for all the symmetries as they have different $\beta_{\mathrm{PR}}$ and $\gamma_{\mathrm{PR}}$ dependencies. Fig. 1a shows a plot comparing $\kappa$ and $\zeta$ for the different symmetries. For the POST element, the scaling factor, $\kappa$, is related to the symmetry numbers $N$ and $n$ by

$|\kappa|=\frac{4(N / n)^{3} \sin (\pi n / N)}{\sqrt{2} \pi\left(16(N / n)^{2}-1\right)}$.

For this element, the r.f. nutation frequency, $\omega_{\mathrm{rf}}$, of the sequence is given by

$\frac{\omega_{\mathrm{rf}}}{\omega_{\mathrm{r}}}=2 \frac{N}{n}$.

Hence, Fig. 1a includes a scale of $\omega_{\mathrm{rf}} / \omega_{\mathrm{r}}$ as well as that of $\kappa$. Unique among the symmetries tested, $\mathrm{C}_{2}^{1}$ allows terms in $\bar{H}_{\mathrm{D}, \mathrm{IS}}^{(2)}$ which are not proportional to $S_{Z}$, giving rise to a large value of $\zeta$.

Fig. 1 includes SPC5 [23], which uses the $\mathrm{C}_{2}^{1}$ symmetry and the POST element. Because $\mathrm{C}_{2}^{1}$ does not exclude all first-order CSA terms from the average Hamiltonian, an $X \bar{X}$ supercycle is used. This also removes those parts of $\bar{H}_{\mathrm{D} \text {.IS }}^{(2)}$ not proportional to $S_{Z}$. We have therefore applied this supercycle to POST-C7 to create a sequence we call SPC7, equivalent to POST-C7 POST-C7. This sequence is also included in Fig. 1.

In general, large $\kappa$ and small $\zeta$ are preferred, such as for POST-C $8_{2}^{1}$. However, lower r.f. amplitudes are also useful. Although sequences such as POST-C20 $0_{6}^{3}$ have smaller values of $\kappa$ than POST-C 82 , the reduction in r.f. power may allow faster spinning. This would improve the overall experimental performance and would also reduce the size of $\bar{H}_{\mathrm{D}, \mathrm{IS}}^{(2)}$, since it is proportional to $b_{\mathrm{IS}}^{2} / \omega_{\mathrm{r}}$.

To account for the effects of ${ }^{1} \mathrm{H}-{ }^{1} \mathrm{H}$ dipolar couplings and $J$-couplings, we have simulated double-quantum filtering (2QF) using the SIMPSON program [24]. The model spin system was constructed from two ${ }^{13} \mathrm{C}$ nuclei and two ${ }^{1} \mathrm{H}$ nuclei arranged in an L-shape, as show in the inset to Fig. $1 \mathrm{~b}$. The ${ }^{13} \mathrm{C}$ parameters (chemical shift, CSA, dipolar couplings and $J$-couplings) were taken from glycine $[25,26]$, whilst the shortest ${ }^{1} \mathrm{H}-{ }^{13} \mathrm{C}$ distances were set to $1.15 \AA$ with a corresponding $J$ coupling of $150 \mathrm{~Hz}$. The MAS frequency was constant at $12 \mathrm{kHz}$. Fig. $1 \mathrm{~b}$ shows the $2 \mathrm{QF}$ efficiency, simulated for the same pulse sequences as in Fig. 1a, plotted against $\kappa / \zeta$. A relatively good correlation between the maximum simulated efficiency and $\kappa / \zeta$ can be seen, with a curve being added to the graph as a guide to the eye. POST-C7 stands out as being better than expected from the value of $\kappa / \zeta$. This may be due to the lesser impact of the off-diagonal terms in $\bar{H}_{\mathrm{D}, \mathrm{IS}}^{(1)}$ on the double-quantum excitation.

From Fig. 1, we would expect to see reasonable performance from POST-C7 without decoupling but we would also expect sequences such as POST-C $20_{6}^{3}$ to perform better, despite using slightly lower r.f. power. Furthermore, SPC7 would be expected to be superior to all other sequences with an equal or lower r.f. power. In contrast, the sequence POST-C 2076 would be expected to perform poorly, despite using the same r.f. power as POST-C $200_{6}^{3}$.

\section{Experimental results and discussion}

We have carried out a series of $2 \mathrm{QF}$ experiments on a $600 \mathrm{MHz}$ wide-bore instrument (Bruker Biospin/Germany) using a standard $2.5 \mathrm{~mm}$ MAS probe. First, we measured, at three different MAS frequencies, the $2 \mathrm{QF}$ efficiency, using POST-C7, as a function of ${ }^{1} \mathrm{H}$ decoupling nutation frequency (Fig. 2). At all three MAS frequencies, $2 \mathrm{QF}$ signal is observed with zero and high ${ }^{1} \mathrm{H}$ decoupling. There is a broad minimum in the intermediate regime, which moves to higher nutation frequencies with higher MAS frequencies. As a result, at 12 $\mathrm{kHz}$ MAS, the $2 \mathrm{QF}$ efficiency is higher for no decoupling than for $120 \mathrm{kHz}$ decoupling.

Fig. 3a shows experimental 2QF build-up curves, obtained on $\left[{ }^{13} \mathrm{C}_{2}\right]$ glycine, for five experimental schemes that may be split into two categories. The first, referred to as HP-DEC, has a low MAS frequency $(7 \mathrm{kHz})$ and a high ${ }^{1} \mathrm{H}$ decoupling nutation frequency $(105 \mathrm{kHz})$. The second, referred to as NO-DEC, has a higher MAS frequency $(12 \mathrm{kHz})$ and no ${ }^{1} \mathrm{H}$ decoupling. To demonstrate the HP-DEC type of experiment, we used POSTC7, whilst for the NO-DEC type of experiment, four of the above-mentioned sequences were used, POST-C7, SPC7, POST-C $20_{6}^{3}$ and POST-C $20_{6}^{7}$. Both approaches lead to similar build-up characteristics with some additional damping for the NO-DEC conditions. The maximum for the NO-DEC experiments is around $500 \mu \mathrm{s}$, whilst it is around $600 \mu$ s for the HP-DEC experiment. Of the five experiments, HP-DEC POST-C7 gives the most signal. NO-DEC POST-C7, SPC7 and POST-C20 ${ }_{6}^{3}$ 


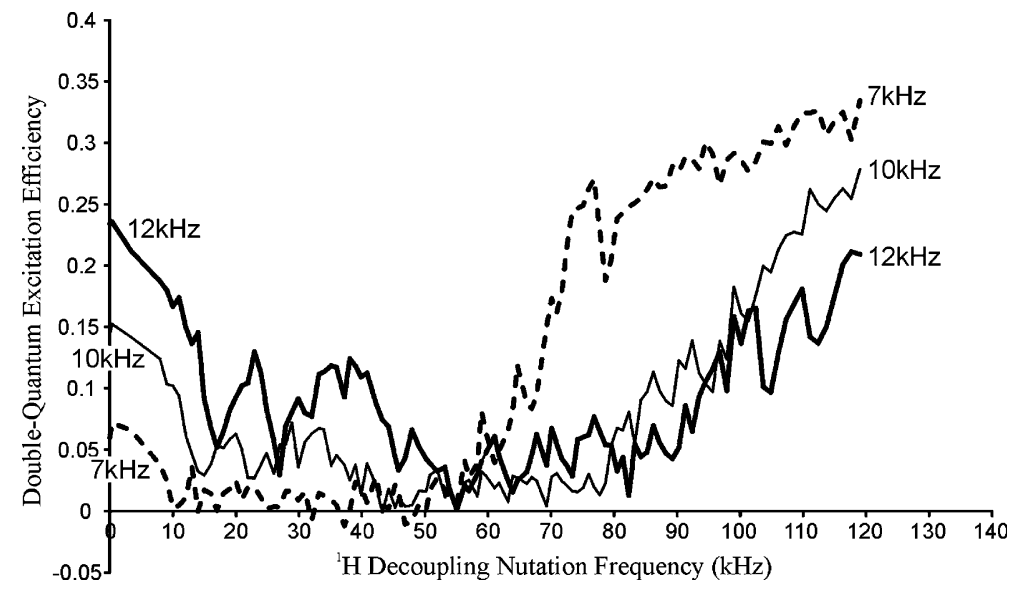

Fig. 2. Experimental POST-C7 2QF efficiency for $\left[{ }^{13} \mathrm{C}_{2}\right]$ glycine as a function of ${ }^{1} \mathrm{H}$ decoupling nutation frequency at three different MAS frequencies, $7 \mathrm{kHz}$ (dashed line), $10 \mathrm{kHz}$ (thin solid line) and $12 \mathrm{kHz}$ (thick solid line). Double-quantum excitation times (equal to reconversion times) were 0.53 $\mathrm{ms}, 0.4 \mathrm{~ms}$ and $0.3 \mathrm{~ms}$, respectively.
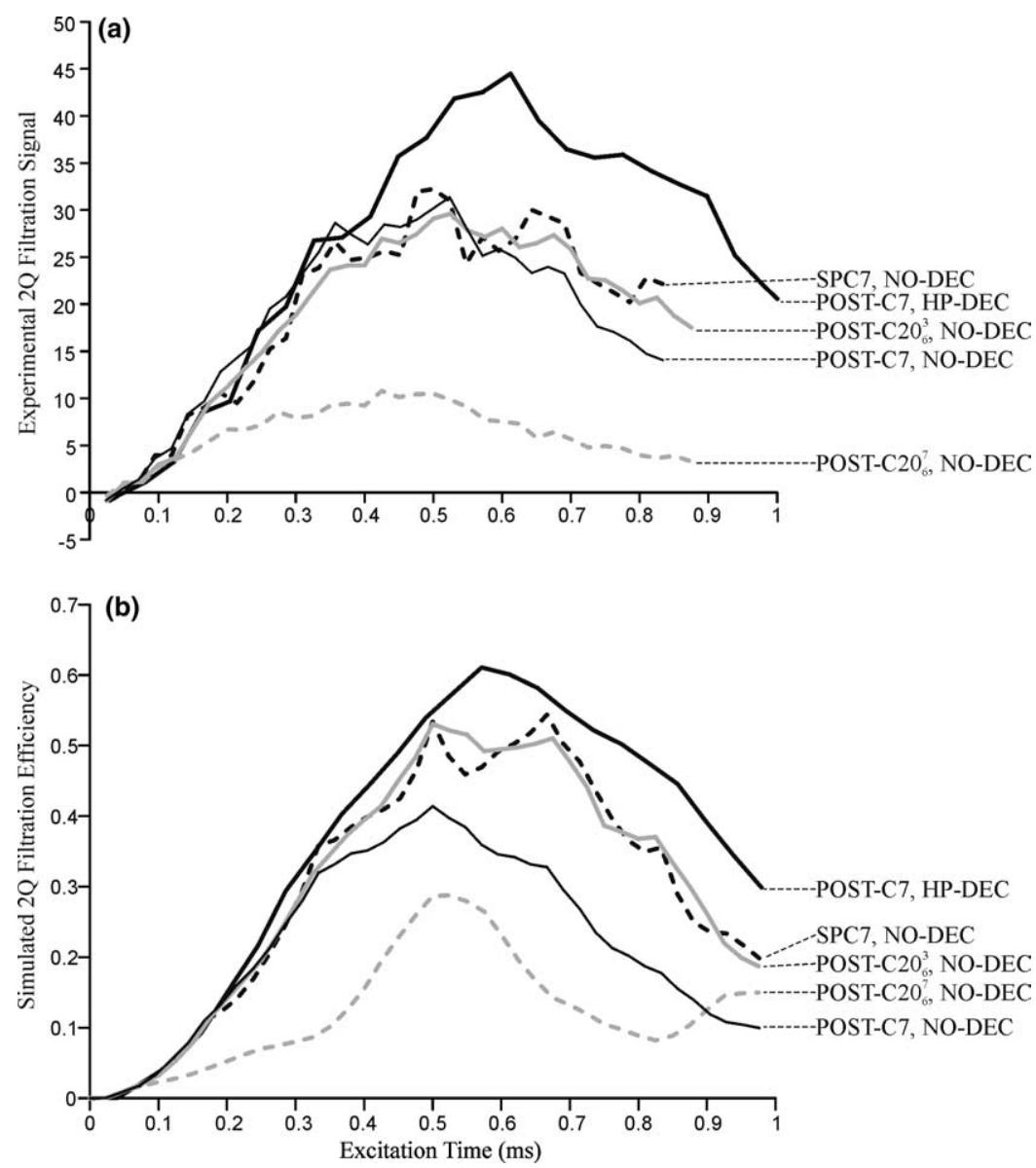

Fig. 3. (a) Experimental $2 \mathrm{QF}$ build-up curves of $\left[{ }^{13} \mathrm{C}_{2}\right]$ glycine. The signal intensity is given in arbitrary units. (b) Simulated $2 \mathrm{QF}$ efficiency build-up curves of a ${ }^{1} \mathrm{H}-{ }^{13} \mathrm{C}-{ }^{13} \mathrm{C}-{ }^{1} \mathrm{H}$ spin system, as described in the text. In both plots, the thick black lines represent POST-C7 HP-DEC, thin black lines represent POST-C7 NO-DEC, dashed black lines represent SPC7 NO-DEC, solid grey lines represent POST-C20 66 NO-DEC and dashed grey lines represent POST-C20 76 NO-DEC. The HP-DEC and NO-DEC conditions are defined in the text.

are relatively similar to each other and give maxima at approximately $70 \%$ of that for the HP-DEC scheme. The expected improvement for SPC7 and POST-C20 $\frac{3}{6}$ over POST-C7 is not observed, although the damping of the signal is somewhat less, suggesting that these sequences may be better suited to recoupling more distant 
(a)

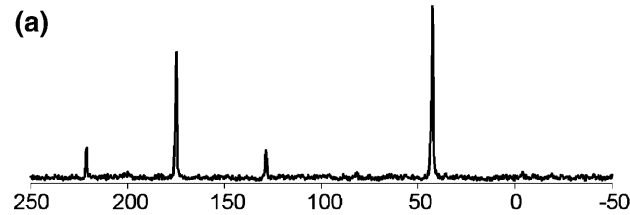

(b)

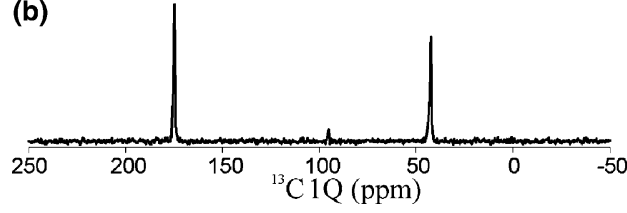

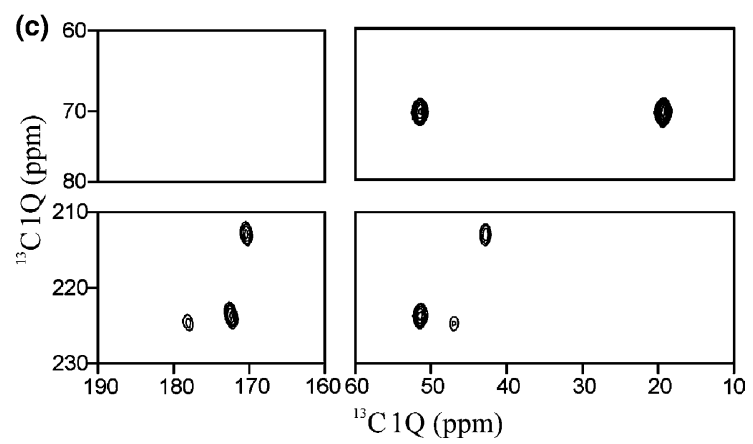

Fig. 4. (a) POST-C7 2QF spectrum of $\left[{ }^{13} \mathrm{C}_{2}\right]$ glycine at $7 \mathrm{kHz}$ MAS with $105 \mathrm{kHz}$ decoupling. Excitation and reconversion times were $530 \mu \mathrm{s}$. (b) SPC7 $2 \mathrm{QF}$ spectrum of $\left[{ }^{13} \mathrm{C}_{2}\right]$ glycine at $12 \mathrm{kHz}$ MAS with no decoupling. Excitation and reconversion times were $500 \mu \mathrm{s}$. (c) POST-C7 (2Q,1Q) 2D correlation spectrum of $\mathrm{U}-\left[{ }^{13} \mathrm{C},{ }^{15} \mathrm{~N}\right] \mathrm{AGG}$ at $12 \mathrm{kHz}$ MAS with no decoupling. Excitation and reconversion times were $333 \mu$ s. 256 increments in $t_{1}$ were acquired, with a total acquisition time of $4.6 \mathrm{~h}$.

spins, when longer excitation and reconversion times are needed. As predicted in Fig. 1, POST-C207 performs very poorly.

Fig. $3 b$ shows simulated build-up curves for the same five experimental schemes, using the same arrangement of two ${ }^{13} \mathrm{C}$ and two ${ }^{1} \mathrm{H}$ nuclei as used for Fig. 1b. The match to the shapes of the experimental build-up curves for NO-DEC POST-C7, SPC7 and POST-C $20_{6}^{3}$ are good but the relative intensities are not as observed experimentally. Although NO-DEC POST-C $20_{6}^{7}$ performs poorly in the simulation, there is not a good match to the shape of the experimental curve, indicating that the simple model for the ${ }^{1} \mathrm{H}$ nuclei is not good enough for a case where the first-order heteronuclear term is large. As is expected, the shape of the HP-DEC POST-C7 simulation matches the experimental curve well, since the ${ }^{1} \mathrm{H}$ nuclei can be expected to play a minimal role here.
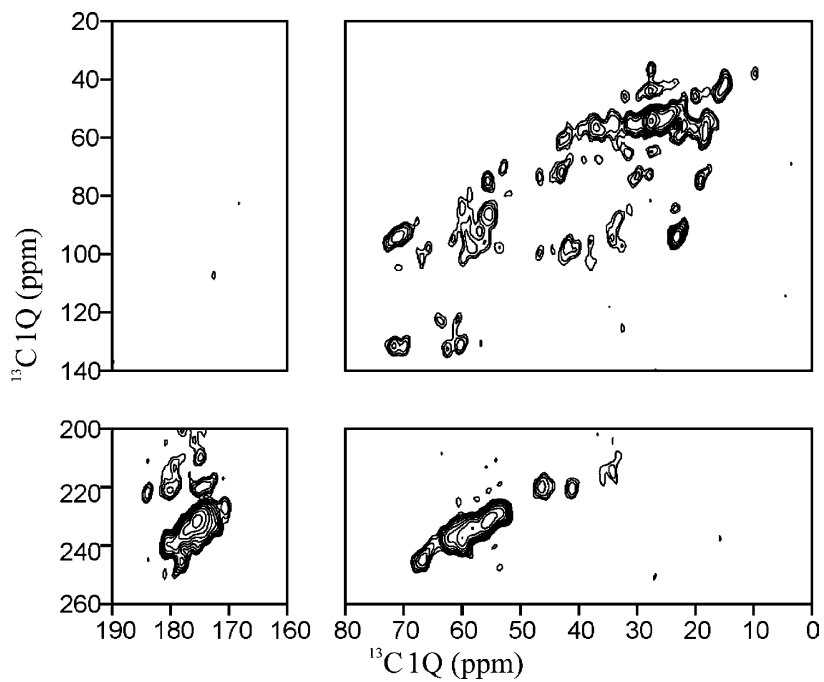

Fig. 5. POST-C20 20 (2Q,1Q) $2 \mathrm{D}$ correlation spectrum of $\mathrm{U}$ $\left[{ }^{13} \mathrm{C},{ }^{15} \mathrm{~N}\right]$ ubiquitin at $12 \mathrm{kHz}$ MAS with no decoupling. Excitation and reconversion times were $525 \mu \mathrm{s} .128$ increments in $t_{1}$ were acquired, with a total acquisition time of $9.5 \mathrm{~h}$.
Fig. 4 shows results of the $1 \mathrm{D} 2 \mathrm{QF}$ experiment on $\left[{ }^{13} \mathrm{C}_{2}\right]$ glycine using optimized excitation and reconversion times. Here, 2QF spectra are compared for POSTC7 under HP-DEC conditions and SPC7 under NODEC conditions. The latter approach reduces the total r.f. power deposition during double-quantum recoupling by a factor of two without significant loss in signal intensities. In fact, the carbonyl (left-hand) peak is significantly more intense under NO-DEC conditions, partly as a result of more efficient spinning sideband suppression. In Fig. 4c, we demonstrate the use of a NODEC recoupling approach in a standard $(2 \mathrm{Q}, 1 \mathrm{Q}) 2 \mathrm{D}$ correlation experiment, which is, for example, of paramount importance for the assignment of ${ }^{13} \mathrm{C}$ spectra of large biomolecules [27]. In Fig. 4c, all intra-residue correlations expected for $\mathrm{U}-\left[{ }^{13} \mathrm{C},{ }^{15} \mathrm{~N}\right] \mathrm{AGG}$ are easily detected and corroborate the spectral assignments previously obtained [28].

Finally, Fig. 5 illustrates the potential of one of the new schemes discussed above, POST-C20 $0_{6}^{3}$ at $12 \mathrm{kHz}$ MAS with no ${ }^{1} \mathrm{H}$ decoupling, on a $1 \mathrm{mg}$, U- $\left[{ }^{13} \mathrm{C},{ }^{15} \mathrm{~N}\right]-$ labelled sample of the 76 residue protein ubiquitin (VLI Research, Malvern, PA). The (2Q,1Q) 2D correlation spectrum shows that POST-C20 ${ }_{6}^{3}$ without ${ }^{1} \mathrm{H}$ decoupling is capable of exciting double-quantum coherence between all directly bonded ${ }^{13} \mathrm{C}-{ }^{13} \mathrm{C}$ pairs, irrespective of the degree of protonation of the carbon positions.

\section{Conclusions}

We have shown that efficient homonuclear polarization transfer in protonated spin systems is possible at fast MAS rates without ${ }^{1} \mathrm{H}$ decoupling. Such conditions are particularly relevant for ultra-high-field solid-state NMR, where fast MAS is necessary to suppress anisotropic chemical shielding and unwanted dipolar recoupling effects. Under these conditions, double-channel, high-power r.f. irradiation may be replaced by the presented NO-DEC dipolar recoupling approach. Our 
results, obtained with the POST-C7, SPC7 and POST$\mathrm{C} 20_{6}^{3}$ recoupling schemes, provide a practical basis for further methodological extensions, which, for example, employ slow phase modulations and low decoupling fields in concert with fast MAS to further improve the details of polarization transfer among rare spins for MAS frequencies above $10 \mathrm{kHz}$. Since the removal of ${ }^{1} \mathrm{H}$ decoupling significantly reduces the total r.f. power used, we expect the presented concept to be particularly useful for biomolecular applications.

\section{Acknowledgements}

This work was funded in part by an Alexander-vonHumboldt post-doctoral fellowship for C.E.H. and the DFG.

\section{References}

[1] E.R. Andrew, A. Bradbury, R.G. Eades, Nature 182 (1958) 1659.

[2] R. Tycko, G. Dabbagh, Chem. Phys. Lett. 173 (1990) 461.

[3] D.M. Gregory, D.J. Mitchell, J.A. Stringer, S. Kiihne, J.C. Shiels, J. Callahan, M.A. Mehta, G.P. Drobny, Chem. Phys. Lett. 246 (1995) 654.

[4] A.E. Bennett, D.P. Weliky, R. Tycko, J. Am. Chem. Soc. 120 (1998) 4897

[5] M. Carravetta, M. Edén, O.G. Johannessen, H. Luthman, P.J.E. Verdegem, J. Lugtenburg, A. Sebald, M.H. Levitt, J. Am. Chem. Soc. 123 (2001) 10628.

[6] Y.K. Lee, N.D. Kurur, M. Helmle, O.G. Johannessen, N.C. Nielsen, M.H. Levitt, Chem. Phys. Lett. 242 (1995) 304.

[7] P.R. Costa, J.D. Gross, M. Hong, R.G. Griffin, Chem. Phys. Lett. 280 (1997) 95.
[8] M. Hong, J.D. Gross, R.G. Griffin, J. Phys. Chem. B 101 (1997) 5869.

[9] R.G. Griffin, Nat. Struct. Biol. 5 (1998) 508.

[10] M. Baldus, Prog. Nucl. Magn. Reson. Spectrosc. 41 (2002) 1.

[11] S. Luca, H. Heise, M. Baldus, Acc. Chem. Res. 36 (2003) 858.

[12] Y. Ishii, J. Ashida, T. Terao, Chem. Phys. Lett. 246 (1995) 439.

[13] M.H. Levitt, in: R.K. Harris (Ed.), Encyclopedia of Nuclear Magnetic Resonance: Supplementary Volume, Wiley, Chichester, UK, 2002, pp. 165-196.

[14] A. Brinkmann, M. Edén, M.H. Levitt, J. Chem. Phys. 112 (2000) 8539.

[15] P.E. Kristiansen, D.J. Mitchell, J.N.S. Evans, J. Magn. Reson. 157 (2002) 253.

[16] R. Verel, M. Baldus, M. Nijman, J.W.M. van Os, B.H. Meier, Chem. Phys. Lett. 280 (1997) 31.

[17] R. Verel, M. Baldus, M. Ernst, B.H. Meier, Chem. Phys. Lett. 287 (1998) 421.

[18] M. Baldus, B.H. Meier, J. Magn. Reson. A 121 (1996) 65.

[19] U. Haeberlen, in: J.S. Waugh (Ed.), Advances in Magnetic Resonance, Academic Press, New York, 1976.

[20] M. Hohwy, H.J. Jakobsen, M. Edén, M.H. Levitt, N.C. Nielsen, J. Chem. Phys. 108 (1998) 2686.

[21] M. Hohwy, N.C. Nielsen, J. Chem. Phys. 109 (1998) 3780.

[22] V.B. Cheng, H.H. Suzukawa, M. Wolfsberg, J. Chem. Phys. 59 (1973) 3992.

[23] M. Hohwy, C.M. Rienstra, C.P. Jaroniec, R.G. Griffin, J. Chem. Phys. 110 (1999) 7983.

[24] M. Bak, J.T. Rasmussen, N.C. Nielsen, J. Magn. Reson. 147 (2000) 296.

[25] P.-G. Jönsson, Å. Kvick, Acta. Cryst. B 28 (1972) 1827.

[26] R.A. Haberkorn, R.E. Stark, H. van Willigen, R.G. Griffin, J. Am. Chem. Soc. 103 (1981) 2534.

[27] S. Luca, J.F. White, A.K. Sohal, D.V. Filipov, J.H. van Boom, R. Grisshammer, M. Baldus, Proc. Nat. Acad. Sci. USA 100 (2003) 10706.

[28] S. Luca, D.V. Filippov, J.H. van Boom, H. Oschkinat, H.J.M. de Groot, M. Baldus, J. Biomol. NMR 20 (2001) 325. 